\title{
Accurate stellar masses in the multiple system T Tauri
}

\author{
G. Duchêne ${ }^{1}$, H. Beust ${ }^{1}$, F. Adjali ${ }^{1}$, Q. M. Konopacky ${ }^{2}$, and A. M. Ghez ${ }^{2}$ \\ ${ }^{1}$ Laboratoire d'Astrophysique de Grenoble, BP 53, 38041 Grenoble Cedex 9, France \\ e-mail: gaspard.duchene@obs.ujf-grenoble.fr \\ 2 Department of Physics and Astronomy, UCLA, Los Angeles, CA 90095-1562, USA
}

Received 27 June 2006 / Accepted 26 July 2006

\section{ABSTRACT}

\begin{abstract}
Aims. We obtain accurate estimates for the individual masses of the components of the tight binary system T Tau $\mathrm{S}$ to settle the ongoing debate on the nature of $\mathrm{T} \mathrm{Tau} \mathrm{Sa,} \mathrm{a} \mathrm{so-called} \mathrm{infrared} \mathrm{companion.}$

Methods. We take advantage of the fact that T Tau S belongs to a triple system composed of two hierarchical orbits to simultaneously analyze the motion of T Tau Sb in the rest frames of T Tau Sa and T Tau N. With this method, it is possible to pinpoint the location of the center of mass of T Tau S and, thereby, to determine individual masses for T Tau Sa and T Tau Sb with no prior assumption about the mass/flux ratio of the system. This improvement over previous studies of the system results in much better constraints on orbital parameters.

Results. We find individual masses of $2.73 \pm 0.31 M_{\odot}$ for T Tau Sa and of $0.61 \pm 0.17 M_{\odot}$ for T Tau Sb (in agreement with its early-M spectral type), including the uncertainty on the distance to the system. These are among the most precise estimates of the mass of any Pre-Main Sequence star, a remarkable result since this is the first system in which individual masses of T Tauri stars can be determined from astrometry only. This model-independent analysis confirms that T Tau Sa is an intermediate-mass star, presumably a very young Herbig Ae star, that may possess an almost edge-on disk.
\end{abstract}

Key words. stars: individual: T Tau - stars: pre-main sequence - binaries: close - astrometry

\section{Introduction}

The most robust method to determine the mass of stars is to monitor the Keplerian orbital motion of multiple systems. This method is particularly valuable for contracting Pre-Main Sequence (PMS) stars, such as T Tauri stars (TTS), for which mass estimates from evolutionary models can differ by factors as large as 2 (e.g., Baraffe et al. 2002). However, there are only about 20 individual TTS with well-determined masses, all of them having radial velocity curves or a Keplerian circumstellar disk (see Hillenbrand \& White 2004 for a recent review).

The triple system T Tauri is among the best-studied PMS multiple systems (see, e.g., Beck et al. 2004). The optically bright $\mathrm{T}$ Tau N, a $\sim 2 M_{\odot}, \sim 1$ Myr-old star, is associated with the infrared(IR)-bright T Tau S tight binary system, which was resolved less than a decade ago (Koresko 2000). The latter consists of T Tau Sb, a deeply embedded, early M-type TTS, and of $\mathrm{T}$ Tau Sa, an extremely red and highly variable object (Ghez et al. 1991) that has been classified as an infrared companion (IRC). Despite two decades of steady observations, the nature of that third component remains largely unknown. It shares many spectral properties with heavily embedded, Class I protostars, but its physical association with two normal TTS seems to contradict this hypothesis if the system is coeval (Koresko et al. 1997). Alternatively, Ghez et al. (1991) proposed that T Tau Sa, which underwent a strong outburst, is a low-mass FU Ori object. More recently, it has been suggested that $\mathrm{T} \mathrm{Tau} \mathrm{Sa}$ is an intermediatemass PMS star surrounded by an opaque edge-on circumstellar disk (Duchêne et al. 2005).

Determining the mass of T Tau Sa from its orbital motion within this triple system would help our understanding of its exact nature. While it has been suggested that this system may be on an unbound orbit (Loinard et al. 2003), other studies have found possible closed orbits and derived a total mass of 2-6 $M_{\odot}$ for T Tau S (Johnston et al. 2003; Beck et al. 2004). Using a refined method, Johnston et al. (2004a,b) attempted for the first time to determine the individual masses of T Tau Sa and Sb. All of these studies suggested that the IRC is the most massive component of the system. However, they were based on simplifying (and sometimes invalid) assumptions regarding the flux ratio and/or mass ratio of the T Tau $\mathrm{S}$ system, resulting in additional sources of uncertainty.

Here, the combined orbital motion of the triple system is revisited using an almost assumption-free, generic approach, in order to obtain a precise estimate of the individual stellar masses within the T Tau S tight system. This Letter is composed as follows: the fitting method and the observations used in this analysis are presented in Sect. 2; the results and implications are discussed in Sect. 3.

\section{Approach and astrometric dataset}

Two types of astrometric data are available for the T Tau multiple system. The first type is high-resolution IR data, which resolves all three components in data obtained since 1997. The second type is radio data, which detected only two of the components for over two decades, T Tau N and a southern source. Although this southern source is shown by Loinard et al. (2003) to be physically associated with T Tau Sb, Smith et al. (2003) and Johnston et al. (2004a) argue that it might be offset from it by up to $0 .{ }^{\prime} 01-0{ }^{\prime} 03$. In our analysis, we assume that the radio and IR source are indeed the same object. Only a much 
longer IR follow-up of the system would allow one to determine whether this assumption is valid.

\subsection{Fitting method}

Two general approaches have been used in previous attempts to fit the orbital motion of the T Tau Sa-Sb tight binary system. First, it is possible to consider in the fit only the IR datasets, which provide the location of both components. In this case, however, the short timespan probed since 1997 poorly constrains the orbit and a wide range of total system masses is allowed (Beck et al. 2004). Including our most recent IR measurements (see Sect. 2.2) only slightly improves this conclusion.

In a second approach, the separation between $\mathrm{T}$ Tau $\mathrm{N}$ and $\mathrm{T}$ Tau Sb, estimated from the much longer radio observations, is transformed into a $\mathrm{T} \mathrm{Tau} \mathrm{Sa}-\mathrm{Sb}$ separation prior to fitting the orbital motion. In this case, one has to make some assumptions regarding the unknown location of $\mathrm{T}$ Tau Sa with respect to $\mathrm{T}$ Tau N. In the past, two main categories of assumptions were made. First, some authors considered that T Tau Sa follows a constant linear motion with respect to T Tau N (Loinard et al. 2003; Johnston et al. 2003), implicitly assuming that it is coincident with the center of mass of T Tau S. However, while T Tau Sa is indeed more massive than its companion, the mass of the latter is not negligible (see Sect. 3). Second, it was assumed that the historical location of the photocenter of T Tau S, which has been observed for over 15 years, can be used to trace the location of T Tau Sa prior to 1997 (Johnston et al. 2004a,b). This requires one to assume that the IR flux ratio within the T Tau $\mathrm{S}$ system remains constant. Evidence for large variability in at least one of the two components of the system is growing, however (Ghez et al. 1991; Beck et al. 2004).

To limit as much as possible the number of assumptions in the analysis, we decided to fit simultaneously two types of data, namely the location of T Tau Sb in two rest frames: with respect to T Tau Sa and to T Tau N. We did not use the separation between T Tau N and T Tau Sa because it would not be independent of the other two types of measurements.

We assume that T Tau Sb follows a bound Keplerian orbit around T Tau Sa and use the $141.5 \pm 2.8 \mathrm{pc}$ distance to the system estimated by Loinard et al. (2005). We further assume that the motion of the center of mass of T Tau S, which is a Keplerian orbit around T Tau N, can be approximated by a parabolic motion over the 20 years of observations probed here. This is justified as the period of this outer orbit is at least 400 years (Johnston et al. 2004a,b). Fitting the motion of T Tau Sb in both rest frames simultaneously allows us to determine individual masses from astrometry only, as the ratio of the amplitude of the motion of $\mathrm{T} \mathrm{Tau} \mathrm{Sb}$ in both frames is a direct measurement of the mass ratio.

We use a Levenberg-Marquardt $\chi^{2}$ minimization routine (Press et al. 1992) that simultaneously fits for 14 independent parameters. The T Tau $\mathrm{Sa}-\mathrm{Sb}$ orbit is described by 7 parameters: semi-major axis $(a)$, eccentricity $(e)$, inclination with respect to the plane of the sky $(i)$, position angle of ascending node $(\Omega$, measured Eastward from North), azimuth of periastron $(\omega)$, time of periastron passage $\left(t_{\mathrm{P}}\right)$ and orbital period $(P)$. The parabolic motion of the center of mass of T Tau $S$ with respect to $\mathrm{T}$ Tau $\mathrm{N}$ is described by 6 parameters defined by its right ascension $\alpha_{\mathrm{S}}=\alpha_{0}+v_{\alpha}\left(t-t_{0}\right)+a_{\alpha}\left(t-t_{0}\right)^{2}$ and a corresponding equation for its declination, with $t_{0}=2000.0$. Finally, the last parameter describes the mass ratio in the system: $\mu=$ $M_{\mathrm{Sb}} / M_{\mathrm{S}}$, where $M_{\mathrm{Sb}}$ and $M_{\mathrm{S}}$ are the mass of T Tau Sb and of the whole T Tau S system, respectively. Kepler's third law is used to determine $M_{\mathrm{S}}$ from the values of $a$ and $P$; the individual stellar masses $M_{\mathrm{Sa}}$ and $M_{\mathrm{Sb}}$ are derived from the values of $M_{\mathrm{S}}$ and $\mu$. The minimization routine was run with several tens of thousand initial guesses spanning wide ranges for all fit parameters to ensure convergence on the absolute minimum of the $\chi^{2}$ function.

The uncertainties on the fit parameters are estimated using a bootstrap method, a robust method when performing a fit with many (correlated) parameters. We created 10000 artificial datasets containing as many points as the observed dataset, in which each point is randomly drawn from a Gaussian distribution centered on the actual measurement and whose width is given by the associated astrometric uncertainty, and run the $\chi^{2}$ minimization routine for each realization. The resulting distribution of values for each fit parameter is characterized by a mean value equal to that of the best fit and by a standard deviation that we consider as the estimate of the uncertainty on the fit parameter.

Because this fitting method is based on a fit of all available measurements without further transformation, and because no parameter is set to an ad hoc fixed value, it is very robust and essentially assumption-free.

\subsection{IR astrometric dataset}

A dozen high spatial resolution IR observations of the T Tau triple system have been published; they usually simultaneously provide the position of $\mathrm{T} \mathrm{Tau} \mathrm{Sb}$ with respect to both other stars. All astrometric measurements listed in Beck et al. (2004) and Duchêne et al. (2005) are included in our fit ${ }^{1}$. Two additional observations of the system were recently published by Mayama et al. (2006). However, they are significantly offset $(\gtrsim 3 \sigma)$ from all other quasi-contemporaneous measurements; these measurements are likely biased or suffer from underestimated uncertainties and they have been discarded from the analysis.

In addition to these measurements, we present new $K$-band speckle interferometric datasets obtained on the $10 \mathrm{~m}$ Keck I telescope in 1997, 2004 and 2005. For the last two epochs, the $\mathrm{T}$ Tau Sa-Sb system is sufficiently well-resolved that the relative astrometry of all components could be determined from shiftand-add images. On the other hand, in 1997, the system was close to the diffraction limit $(\approx 1.25 \lambda / D)$ and traditional speckle analysis was conducted. The usual basic reduction techniques were first applied to all datasets (see Ghez et al. 1993 for more details). Because T Tau is a hierarchical triple system, a 2-step method identical to that described in Duchêne et al. (2003) was used to extract its properties. The new astrometric and photometric data are presented in Table 1.

\subsection{Radio astrometric dataset}

Loinard et al. (2003) and Johnston et al. (2003, 2004b) have published a series of VLA centimeter observations of T Tau spanning 20 years, from 1983 to 2003. Both groups independently analyzed the 1983, 1988, 1992, 1995 and 2001 datasets, and obtained very similar results. We found that using either sets of results for these epochs yields very similar fits ${ }^{2}$. In the following, we use the $2 \mathrm{~cm}$ astrometric results from Johnston et al. (2003, 2004b) in order to ensure a maximum uniformity among the

\footnotetext{
The astrometric measurements presented in Duchêne et al. (2002) suffered from an incorrect astrometric calibration: the position angles used in this study are $1.1^{\circ}$ larger than was previously reported.

2 It is however incorrect to simultaneously fit both, as was done by Tamazian (2004), since they come from the same raw datasets.
} 

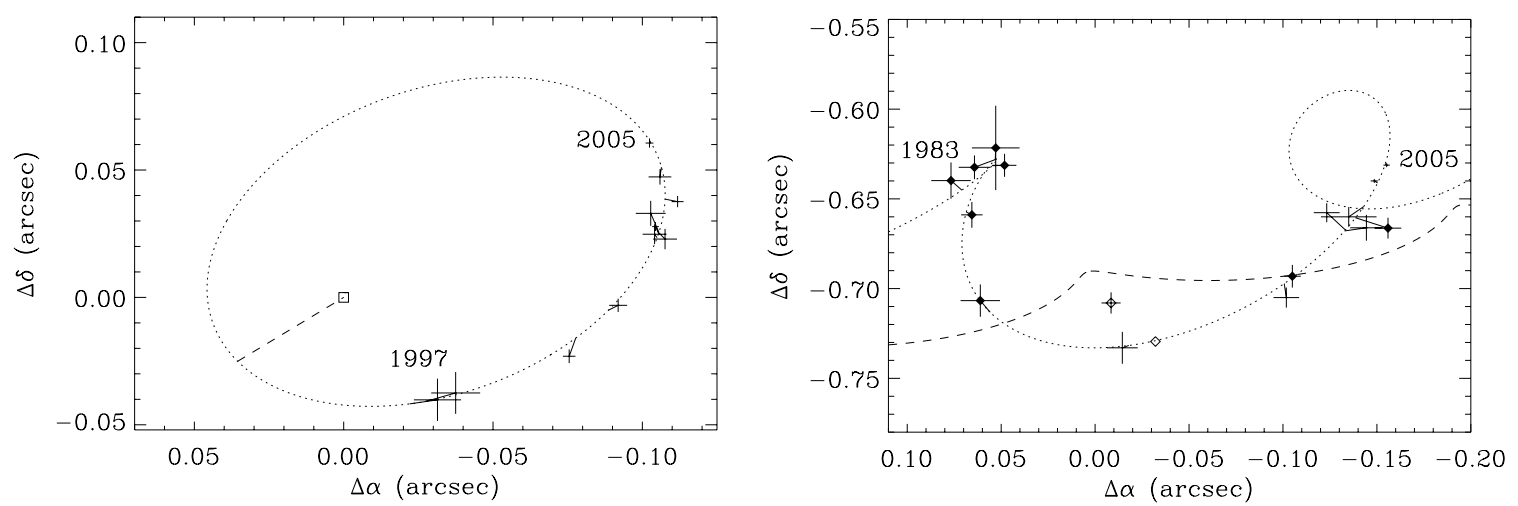

Fig. 1. Observed motion of T Tau Sb in the reference frame of T Tau Sa (left $)$ and $\mathrm{T}$ Tau $\mathrm{N}($ right). The dotted curves represent our best combined fit; each observation is connected to its modeled counterpart by a straight line. In the left-hand panel, the square indicates the fixed location of $\mathrm{T} \mathrm{Tau} \mathrm{Sa}$ and the dashed line connects it to the periastron. In the right-hand panel, filled diamonds represent the VLA measurements; the open diamonds indicate the 1998 VLA measurement excluded from the final fit and its fitted counterpart. In this panel, the dashed curve represents the motion of $\mathrm{T} \mathrm{Tau} \mathrm{Sa}$.

Table 1. New astrometric and photometric data for the T Tau system.

\begin{tabular}{cccc}
\hline \hline UT Date & Sep. $\left(^{\prime \prime}\right)$ & PA $\left(^{\circ}\right)$ & $\Delta K$ \\
\hline \multicolumn{4}{c}{ T Tau Sa-Sb } \\
\hline 1997 Oct. 12 & $0.051 \pm 0.009$ & $218 \pm 8$ & $3.0 \pm 0.1$ \\
2004 Dec. 19 & $0.116 \pm 0.004$ & $294.1 \pm 1.4$ & $0.76 \pm 0.12$ \\
2005 Nov. 13 & $0.119 \pm 0.001$ & $300.6 \pm 1.0$ & $0.52 \pm 0.13$ \\
\hline \multicolumn{4}{c}{ T Tau N-Sb } \\
\hline 1997 Oct. 12 & $0.733 \pm 0.009$ & $181.1 \pm 0.6$ & $5.6 \pm 0.2$ \\
2004 Dec. 19 & $0.657 \pm 0.001$ & $193.07 \pm 0.17$ & $3.25 \pm 0.06$ \\
2005 Nov. 13 & $0.650 \pm 0.001$ & $193.81 \pm 0.15$ & $2.42 \pm 0.12$ \\
\hline
\end{tabular}

radio datasets and their analysis, as well as the $19983.7 \mathrm{~cm}$ measurement from Loinard et al. (2003), since no $2 \mathrm{~cm}$ observations were conducted at that epoch.

\section{Results and implications}

Overall, we considered in our fit a total of 11 astrometric measurements of the T Tau Sa-Sb system and 16 measurements of the T Tau N-Sb system. This is the most extensive dataset fitted to date on this system, considering that we did not try to fit the historical location of the photocenter of T Tau $\mathrm{S}$ (but see Sect. 3.1).

\subsection{Individual masses within TTau S}

Fitting all measurements described in Sect. 2, a best fit solution with $\chi_{\text {red }}^{2}=1.88$ is obtained, a slightly large value considering that there are 40 degrees of freedom. In fact, the 1998 VLA measurement reported by Loinard et al. (2003) is $4.1 \sigma$ away from the fitted value and contributes a large amount to the total $\chi^{2}$. All other measurements are within $3 \sigma$ of the fit, and all but three less than $2 \sigma$ away from it, as expected from a Gaussian distribution of the astrometric uncertainties. This particular VLA measurement is the only one included in our fit that was obtained at $3.7 \mathrm{~cm}$. Substantial differences were noted in the past between $2 \mathrm{~cm}$ and $3.7 \mathrm{~cm}$ VLA measurements for this source (Johnston et al. 2003, 2004b), and we therefore consider this point as suspicious.

Excluding the 1998 VLA measurement, a best fit solution which is less than $2.7 \sigma$ away from all remaining measurements and that has a much more acceptable $\chi_{\text {red }}^{2}=1.37$ is obtained with 38 degrees of freedom. No fit parameter is different by more
Table 2. Parameters of the best orbital solution.

\begin{tabular}{cc|cc}
\hline \hline \multicolumn{4}{c}{ Fit parameters } \\
\hline$a\left(10^{-3}{ }^{\prime \prime}\right)$ & $82.1 \pm 1.8$ & $\alpha_{0}\left(10^{-3}{ }^{\prime \prime}\right)$ & $-20.7 \pm 4.0$ \\
$e$ & $0.466 \pm 0.031$ & $\delta_{0}\left(10^{-3}{ }^{\prime \prime}\right)$ & $-694.3 \pm 2.9$ \\
$i\left(^{\circ}\right)$ & $37.2 \pm 5.1$ & $v_{\alpha}\left(10^{-3}{ }^{\prime \prime} / \mathrm{yr}\right)$ & $-9.12 \pm 0.54$ \\
$\Omega\left({ }^{\circ}\right)$ & $115.2 \pm 6.8$ & $v_{\delta}\left(10^{-3} \prime / \mathrm{yr}\right)$ & $1.71 \pm 0.39$ \\
$\omega\left(^{\circ}\right)$ & $12.5 \pm 10.3$ & $a_{\alpha}\left(10^{-4}{ }^{\prime \prime} / \mathrm{yr}^{2}\right)$ & $1.09 \pm 0.50$ \\
$t_{\mathrm{P}}$ & $1995.86 \pm 0.21$ & $a_{\delta}\left(10^{-4}{ }^{\prime \prime} / \mathrm{yr}^{2}\right)$ & $1.18 \pm 0.58$ \\
$P(\mathrm{yr})$ & $21.66 \pm 0.93$ & $\mu$ & $0.183 \pm 0.044$ \\
\hline \multicolumn{4}{c}{ Derived quantities ${ }^{a}$} \\
\hline$A(\mathrm{AU})$ & $11.59 \pm 0.35$ & $M_{\mathrm{S}}\left(M_{\odot}\right)$ \\
$M_{\mathrm{Sa}}\left(M_{\odot}\right)$ & $2.73 \pm 0.31$ & $M_{\mathrm{Sb}}\left(M_{\odot}\right)$ & $3.34 \pm 0.36$ \\
\hline
\end{tabular}

${ }^{a}$ The uncertainty on the derived quantities include that induced by the distance estimate.

than $1.4 \sigma$ from the previous solution, and we consider this new solution as our best fit in the following. The best fit parameters are listed in Table 2 and the solution is illustrated in Fig. 1. Note that it is not possible to distinguish the ascending and descending nodes of the orbit from astrometry only: an identical projected orbit could be described with $\Omega=295.2^{\circ}$ and $\omega=-167.5^{\circ}$.

We then performed a consistency check regarding the historical measurements of the IR photocenter of the T Tau S binary. While strong variability prevents using these measurements in the fit, it must be verified that the photocenter is located along the line joining the fitted location of T Tau Sa and T Tau Sb at all epochs. The photocenter lies within $2.4 \sigma$ of the predicted line connecting the two components for all measurements listed in Ghez et al. (1995) and Roddier et al. (2000), giving more confidence to the validity of the fit.

Including the uncertainty induced by the distance estimate, the total relative uncertainty on $M_{\mathrm{Sa}}$ is about $11 \%$, among the most accurate individual mass estimates for a TTS that does not belong to an eclipsing binary system (Hillenbrand \& White 2004). The uncertainty on $M_{\mathrm{Sb}}$ is about $28 \%$, still a remarkably accurate estimate for a TTS, considering that only astrometric measurements were taken into account in this analysis.

\subsection{Discussion}

The results obtained here are in good agreement with those presented by Johnston et al. (2004a,b), although with uncertainties that are at least half for all parameters: the best estimate for $M_{\mathrm{Sa}}$ 
to date was $2.1 \pm 0.8 M_{\odot}$ (Johnston et al. 2004b). This significant improvement is due to a combination of factors: the increased time coverage of high-resolution IR datasets, a simpler characterization of the T Tau N-S orbit (parabolic motion instead of a Keplerian orbit with several parameters fixed to ad hoc values), the improved distance estimate obtained by Loinard et al. (2005), and the smaller number of assumptions used in our analysis. In this work, the only assumptions that could be disputed are the parabolic approximation of the orbital motion of T Tau S around $\mathrm{T}$ Tau $\mathrm{N}$ over 20 years and the match between the radio and IR location of T Tau Sb. The fact that a very satisfying fit was obtained appears to validate these hypotheses.

The mass derived for $\mathrm{T} \mathrm{Tau} \mathrm{Sb}$ is in excellent agreement with its known, early-M spectral type (Duchêne et al. 2002), as already discussed by Johnston et al. (2004a,b). For the first time, however, an accurate estimate of the mass of T Tau Sa is obtained, leaving little doubt that it is somewhat more massive than $\mathrm{T}$ Tau $\mathrm{N}$ itself and suggesting that T Tau Sa is a young embedded Herbig Ae star. This is the first time that this is determined with such certainty for an IRC. As discussed in Duchêne et al. (2005), it is likely that T Tau Sa is surrounded by a compact and partially opaque circumstellar disk that accounts for the apparent faintness of this intrinsically bright object. Given the orbital parameters of the $\mathrm{T} \mathrm{Tau} \mathrm{Sa}-\mathrm{Sb}$ system, the radius of this disk must be $\lesssim 3 \mathrm{AU}$, i.e., half the periastron distance. This disk must be very inclined with respect to the orbital plane $\left(\Delta i \gtrsim 45^{\circ}\right)$, raising the possibility of gravitational instabilities.

Within about 10 years, spatially-resolved IR observations of the T Tau triple system will allow the derivation of accurate orbital parameters for $\mathrm{T}$ Tau $\mathrm{S}$ without making use of radio datasets. With such an analysis, it will be possible to determine whether the southern radio component of T Tau arises from the chromosphere of $\mathrm{T} \mathrm{Tau} \mathrm{Sb}$ or whether it is a separate object that is simply associated with this object.

In the shorter term, the dynamical stability of the T Tau system must be studied in more detail: the system contains three stars within $100 \mathrm{AU}$ of each other, each of them possessing a circumstellar disk. The overall velocity of $\mathrm{T}$ Tau $\mathrm{S}$ with respect to $\mathrm{TTauN}$ is of the order of $6 \mathrm{~km} \mathrm{~s}^{-1}$, very similar to the velocity of a $100 \mathrm{AU}$-radius circular orbit for a $5 M_{\odot}$ system (assuming $M_{\mathrm{N}}=2 M_{\odot}$ ). This suggests that the outer orbit is seen close to face-on and has a low eccentricity. If this is indeed the case, the application of the stability criterion proposed by Eggleton \& Kiseleva (1995) suggests that the system could be marginally stable. Moreover, the stability of the circumstellar disks is not necessarily ensured in such a tight system. This motivates a follow-up dynamical study similar to that performed for the quadruple system GG Tau (Beust \& Dutrey 2005, 2006) to determine the possible solutions for the outer orbit that preserve the three components and their disks for at least $1 \mathrm{Myr}$, i.e., the age of the system. A preliminary analysis, which will be presented in a forthcoming paper, suggests that the three-body system is stable over a few Myrs, whereas the fate of the circumstellar disks is not yet established.

Acknowledgements. We acknowledge our referee, Mike Simon, for his insightful remarks. We thank J. Lu for her help in calibrating the NIRC camera, L. Saugé and X. Delfosse for clarifying discussions on the methods to derive uncertainties, and L. Loinard for providing his radio measurements. This work was funded in part by the NASA Astrobiology Institute and the NSF Science \& Technology Center for AO, managed by UCSC (AST-9876783). The data presented herein were obtained at the W.M. Keck Observatory, which is operated as a scientific partnership among the California Institute of Technology, the University of California and the National Aeronautics and Space Administration. The Observatory was made possible by the generous financial support of the W.M. Keck Foundation.

\section{References}

Baraffe, I., Chabrier, G., Allard, F., \& Hauschildt 2002, A\&A, 382, 563 Beck, T. L., Schaefer, G. H., Simon, M., et al. 2004, ApJ, 614, 235 Beust, H., \& Dutrey, A. 2005, A\&A, 439, 585

Beust, H., \& Dutrey, A. 2006, A\&A, 446, 137

Duchene, G., Ghez, A. M., \& McCabe, C. 2002, ApJ, 568, 771

Duchene, G., Ghez, A. M., McCabe, C., \& Weinberger, A. J. 2003, ApJ, 592, 288

Duchene, G., Ghez, A. M., McCabe, C., \& Ceccarelli, C. 2005, ApJ, 628, 832

Eggleton, P., \& Kiseleva, L. 1995, ApJ, 455, 640

Ghez, A. M., Neugebauer, G., Gorham, P. W., et al. 1991, AJ, 102, 2066

Ghez, A. M., Neugebauer, G., \& Matthews, K. 1993, AJ, 106, 2005

Ghez, A. M., Weinberger, A. J., Neugebauer, G., et al. 1995, AJ, 110, 753

Hillenbrand, L. A., \& White, R. J. 2004, ApJ, 604, 741

Johnston, K. J., Gaume, R. A., Fey, A. L., et al. 2003, AJ, 125, 858

Johnston, K. J., Fey, A. L., Gaume, R. A., et al. 2004a, ApJ, 604, L65

Johnston, K. J., Fey, A. L., Gaume, R. A., et al. 2004b, AJ, 128, 822

Koresko, C. D. 2000, ApJ, 531, L147

Koresko, C. D., Herbst, T. M., \& Leinert, Ch. 1997, ApJ, 480, 741

Loinard, L., Rodríguez, L. F., \& Rodríguez, M. I. 2003, ApJ, 587, L47

Loinard, L., Mioduszewski, A. J., Rodríguez, L. F., et al. 2005, ApJ, 619, L179

Mayama, S., Tamura, M., Hayashi, M., et al. 2006, PASJ, 58, 375

Press, W. H., Flannery, B. P., Teulosly, S. A., \& Vetterling, W. T. 1992, Numerical Recipes in FORTRAN (Cambridge Univ. Press)

Roddier, F., Roddier, C., Brandner, W., et al. 2000, in Birth and evolution of binary stars, ed. B. Reipurth, \& H. Zinnecker, 60

Smith, K., Pestalozzi, M., Güdel, M., et al. 2003, A\&A, 406, 957

Tamazian, V. S. 2004, AJ, 127, 2378 